\title{
Una red de sensores inalámbricos para la automatización y control del riego localizado
}

\author{
A wireless sensor network for drip irrigation control and automation
}

\section{Nesly Diana Castro C.; Luis Eduardo Chamorro F. ${ }^{2}$; Carlos Andrés Viteri M. ${ }^{3}$}

1 Ingeniera Electrónica, Universidad de Nariño, Grupo de Investigación en Ingeniería Eléctrica y Electrónica (GIIEE), Pasto, Colombia, nesly_castro@yahoo.com

2 Ingeniero Electrónico, Universidad de Nariño, Grupo de Investigación en Cultivos Andinos, Pasto, Colombia, luchofer115@hotmail.com, luisedchaf@hotmail.com.

3 Magíster en Ingeniería Eléctrica, Universidad de Nariño, Grupo de Investigación en Ingeniería Eléctrica y Electrónica (GIIEE), Pasto, Colombia, caviteri@udenar.edu.co

Citar: CASTRO, N.; CHAMORRO, L.; VITERI, C. Una red de sensores inalámbricos para la automatización y control del riego localizado. Rev. Cienc. Agr. 33(1):106-116. doi: http://dx.doi.org/10.22267/rcia.163302.57.

Recibido: Junio 25 de 2015 Aceptado: Agosto 04 de 2016

\section{RESUMEN}

Uno de los objetivos de la agricultura de precisión, es el riego de acuerdo con las necesidades particulares de cada porción del suelo de cultivo. En este trabajo, se describe el diseño e implementación de una red de sensores inalámbricos que es capaz de obtener mediciones de humedad del suelo de diferentes zonas de un cultivo de fresas y, según los datos recogidos, determina el tiempo de riego y la zona particular que se debe regar utilizando el método de goteo. Los requerimientos de agua para los cultivos se estimaron teniendo en cuenta la capacidad de campo y el umbral de riego; ambas fueron encontradas para cada zona del cultivo en periodo de producción. La comunicación entre los nodos de la red se basa en la pila de protocolos ZigBee, y el prototipo final es un sistema de control de riego de encendido/apagado en lazo cerrado, que incluye una banda de histéresis. Los resultados de la implementación de la red y el sistema de control muestran un incremento en la eficiencia del uso de agua, además de una mejora en la calidad de los frutos con respecto al método de riego tradicional.

Palabras clave: agricultura de precisión, ZigBee, humedad volumétrica.

\begin{abstract}
One of the main objectives of precision agriculture is the crops irrigation according to the particular needs of a cropland. This paper describes the design and implementation of a wireless sensor network, which is able to get soil moisture measurements from different zones of a strawberries crop. A control
\end{abstract}


system determines the irrigation time and the particular zone that must be watered by the drip irrigation method, according to the data collected by the network. Water requirements for crops were estimated by field capacity and irrigation threshold; both parameters were calculated for the entire crop in the production stage. The communication between network nodes is based on the ZigBee protocol stack, and the final prototype is a closed-loop on/off control system with a hysteresis band. Finally, the results showed a more water use efficiency and improved strawberry quality when compared to the traditional irrigation system.

Keywords: Precision agriculture, ZigBee, volumetric moisture.

\section{INTRODUCCIÓN}

El objetivo primario de la agricultura de precisión (AP), es la aplicación localizada de insumos agrícolas, de acuerdo con las necesidades específicas de cada porción de suelo en un cultivo. Esta práctica, que incorpora tecnología al campo, está siendo adoptada a nivel mundial ya que permite distribuir de forma eficiente los recursos, incrementar la calidad de los productos y transformar la agricultura en un sector más rentable. Beneficios como éstos son fundamentales en países en desarrollo, donde la agricultura, es uno de los sectores claves para la generación de empleo y por tanto, una de las principales actividades económicas de su población (SAC, 2010).

A pesar de la importancia del sector agrícola, en muchas regiones fértiles de Colombia, aún se mantiene una visión anticuada del campo y se continúan utilizando métodos rudimentarios para su explotación. Esto causa un impacto ambiental y socioeconómico negativo. Las prácticas agrícolas tradicionales, consideran los cultivos como terrenos homogéneos que demandan la misma cantidad de recursos en cada fracción de suelo, razón por la cual, la aplicación de insumos se hace de forma uniforme de acuerdo con los conocimientos empíricos del agricultor.

Definir cuál es la cantidad apropiada aplicable a todo el terreno, no debe ser una decisión subjetiva, menos cuando es posible identificar una variabilidad espacial en el terreno: distintas texturas, niveles de humedad, incidencia de insectos, maleza y otros factores similares (Montavani et al., 2006). Cuando la variabilidad espacial es ignorada y se insiste en la aplicación subjetiva y no localizada de insumos, la productividad del cultivo se ve afectada directamente, ya que los recursos no son distribuidos de forma eficiente y los productos cosechados podrían carecer de la calidad necesaria para ser competitivos en mercados exigentes y selectivos.

Siendo el agua un insumo agrícola limitado y de carácter vital en la agricultura, su distribución óptima es una prioridad (Gonzáles et al., 2012). Actualmente, se instalan distintos métodos de riego carentes de un sistema que en conjunto permita medir la humedad de suelo, controlar el tiempo de riego e identificar la porción de suelo que realmente necesita ser regada (Deaquiz et al., 2014). Esto es una desventaja porque el déficit de riego causa un bajo rendimiento en su producción y, por otro lado, el exceso de riego puede desencadenar infertilidad permanente (Klohn y Appelgren, 1999). En este punto es cuando los conceptos de la AP dejan de ser tan solo una opción tecnológica y se convierten en una necesidad para cubrir de forma precisa la demanda hídrica de un cultivo (Vega et al., 2013).

Este artículo describe el diseño e implementación de una red de sensores inalámbricos que mide continuamente la humedad volumétrica 
del suelo en sitios específicos de un cultivo de fresas. Con esta información, un sistema de control define el tiempo de riego y la sección de suelo que debe ser regada por medio del método de goteo. El sistema descrito se expone como una exploración de pertinencia y beneficios reales, al definir de forma técnica un cronograma de riego bajo conceptos básicos de AP. Además, el sistema permite contrastar los resultados con aquellos obtenidos mediante prácticas de riego no localizadas y tradicionales que son utilizadas tradicionalmente por los pequeños cultivadores de Colombia.

\section{MATERIALES Y MÉTODOS}

\section{Segmentación del Área Cultivable}

Variabilidad del cultivo. Para el desarrollo de éste trabajo, fue seleccionado un cultivo de fresas ubicado en el Altiplano de Pasto, a 2813

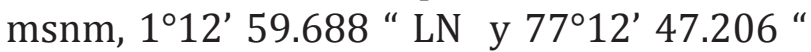
LO al suroccidente colombiano. El área del cultivo correspondió a $600 \mathrm{~m}^{2}(20 \times 30 \mathrm{~m})$ y está sembrado en surcos elevados de doble hilera, cada uno protegido con un recubrimiento plástico acolchado y equipado con dos cintas de goteo laterales que riegan las plantas de forma localizada. El área cultivada se encuentra sobre la ladera de una montaña, con una pendiente aproximada de $12 \%$, los surcos se encuentran paralelos a las curvas de nivel del terreno.

Siguiendo los conceptos de la AP, es preciso identificar la variabilidad del área cultivable, es decir, aquellos factores capaces de alterar considerablemente el desarrollo de las plantas (Mandal y Maity, 2013). La aparente simplicidad de recolectar datos de distintas zonas del terreno o la recaudación de información a partir de la observación detallada del agricultor, no excluyen la posibilidad de determinar el tipo de variabilidad espacial y/o temporal presente en el área cultivable ni mucho menos, de tomar decisiones para distribuir acertadamente los recursos de forma sitio específico. Bajo este contexto el historial de rendimiento del cultivo se evaluó durante su ciclo productivo con el fin de reconocer su variabilidad (Roel y Terra, 2006). Adicionalmente el suelo y su capacidad de retención de agua se examinaron detalladamente de acuerdo con su textura, así como también fueron identificadas las secciones del cultivo que entregaban frutos de mayor y menor calidad durante la cosecha siguiendo el historial de producción. Gracias a esta información se observó una correlación entre el nivel de producción y desarrollo de la planta y la topografía del cultivo que fue considerada para delimitar grupos de surcos o secciones del cultivo con necesidades específicas.

Zonas de manejo. Las diferencias encontradas, se denominan variabilidad espacial de campo. Este hallazgo, permitió concebir el cultivo como un conjunto de secciones o zonas de manejo que requieren dosis diferentes de insumos. El tamaño y cantidad de las zonas en un cultivo, depende principalmente de la capacidad del agricultor de manejar diferencialmente varias secciones $\mathrm{y}$ del tipo de variable de control considerada (Zhang et al., 2002).

Teniendo en cuenta estos factores, el cultivo de fresas fue segmentado en tres zonas de manejo independientes: zona alta, zona media y zona baja, haciendo referencia a la topografía inclinada del terreno. De acuerdo con las evaluaciones realizadas, cada zona se caracterizó por presentar diferencias en cuanto a producción, rendimiento y calidad, que se evidenciaron bajo observación, al evaluar vigor, textura, color y densidad de los frutos en cada zona. Por ejemplo, la zona baja presentaba frutos con textura oscura, carentes de calidad y tamaño, razón por la cual, 
en la mayoría de casos, eran desechados y se optaba por hacer recolección del producto en tiempos distintos a los demás sitios del lote.

\section{Calibración de sensores}

Sensores y surcos guía. La humedad de suelo especifica el contenido de agua que éste retiene en un momento dado, suele definirse en forma volumétrica, cuando resulta de la relación entre el volumen del agua y el volumen total o aparente del suelo (Badillo et al., 2009). Su valor es expresado comúnmente como un porcentaje que puede ser obtenido por métodos directos (cuando incluyen muestreo y análisis de suelo) e indirectos (cuando incluyen sensores instalados cerca a la raíz de las plantas que comunican su nivel hídrico en tiempo real) (Muñoz y Ritter, 2005).

Para medir el nivel de humedad, fue seleccionado en cada zona de manejo un surco guía en el que fue instalado un sensor donde el valor medido por éste, fue asumido como el nivel de humedad promedio actual de la zona asociada (Kuncham y Rao, 2014). Los sensores elegidos fueron los 10HS de Decagon Devices, que entregan una señal de voltaje análoga proporcional a la humedad volumétrica utilizando la técnica de capacitancia o dominio de la frecuencia (FD), y que se destacan por su bajo consumo de potencia y por su amplio volumen de sensibilidad (Decagon, 2009).

El fabricante provee una ecuación para calcular el dato porcentual de humedad volumétrica a partir del voltaje que registra un sensor al ser insertado en un volumen de suelo (Spelman y Kunberger, 2013); sin embargo, para garantizar precisión en la aplicación, se obtuvieron experimentalmente tres ecuaciones para cada zona de manejo y además, una ecuación general para calcular la humedad promedio de todo el cultivo que, posterior a la implementación, permitió evaluar la gestión riego sitio-específico frente a la tradicional .
Muestras de suelo y ecuaciones de calibración. El procedimiento para obtener las ecuaciones consistió en determinar la humedad de suelo a partir del método gravimétrico/ volumétrico recolectando muestras y relacionando estos resultados con los valores de voltaje entregados por los sensores (Cobos y Chambers, 2010). Cuatro muestras fueron tomadas en tubos de PVC de 6 pulgadas; tres no perturbadas extraídas de cada surco guía y una muestra perturbada representativa de todo el cultivo. Esta última formada por varias muestras extraídas de quince surcos distantes (Carreño y Unigarro, 2005).

Una vez recolectadas las muestras, fueron trasladadas y humedecidas hasta saturar su contenido y en el centro de cada una de ellas, fue enterrado verticalmente un sensor $10 \mathrm{HS}$. Partiendo de esta condición inicial, durante 10 días, las muestras de suelo reposaron a la intemperie para lograr un secado natural bajo condiciones climáticas similares a las del cultivo de fresas, así, diariamente fueron medidos el voltaje del sensor y el peso de cada muestra. Transcurrido éste tiempo, las muestras se llevaron a un laboratorio donde fueron secadas y pesadas nuevamente. Finalmente, al relacionar y evaluar todos los datos medidos en ecuaciones propias del área agrícola (Muñoz y Ritter, 2005), se calculó directamente la humedad volumétrica presente en cada muestra y el voltaje real asociado a ese nivel de humedad.

Los datos de cada muestra, fueron organizados en pares ordenados Voltaje - Humedad $\left(\mathrm{V}, \theta_{v}\right)$ para definir una tendencia de comportamiento que pudiera expresarse como una función. Después de aplicar distintos tipos de regresiones, la curva que mejor se ajustó a los diagramas de dispersión descritos, fue la de tipo cuadrática (Mathworks, 2016). Las ecuaciones resultantes se muestran en la Tabla 1. 
Tabla 1. Ecuaciones de calibración para sensores 10HS.

\begin{tabular}{lc}
\multicolumn{1}{c}{ Zona } & Ecuación \\
\hline Alta & $\boldsymbol{\theta}_{v}=\mathbf{1 , 4 6} \times \mathbf{1 0}^{-5} \mathrm{~V}^{\mathbf{2}}+\mathbf{0 , 0 3 1 V}-\mathbf{6}$ \\
Media & $\boldsymbol{\theta}_{v}=\mathbf{3 , 2 0} \times \mathbf{1 0}^{-5} \mathrm{~V}^{2}+\mathbf{0 , 0 1 2 V}-\mathbf{6}$ \\
Baja & $\boldsymbol{\theta}_{v}=\mathbf{2 , 9 7} \times \mathbf{1 0}^{-5} \mathrm{~V}^{2}+\mathbf{0 , 0 1 6 V}-\mathbf{6}$ \\
Cultivo Total & $\boldsymbol{\theta}_{v}=\mathbf{2 , 1 4} \times \mathbf{1 0}^{-5} \mathrm{~V}^{2}+\mathbf{0 , 0 2 6 V}-\mathbf{6}$ \\
\hline
\end{tabular}

\section{Diseño de la red}

Descripción general del diseño. Una red inalámbrica de sensores (WSN, Wireless Sensor Network), es una red de corto alcance, baja velocidad y bajo consumo energético que consiste en un conjunto de nodos autónomos espacialmente distribuidos que monitorean su entorno (Sohraby et al., 2007).

La red diseñada, consta de seis nodos, tres de ellos son nodos sensores, dos son nodos actuadores y el principal es el coordinador de la red. Todos ellos fueron instalados con una topología en estrella en la que cada nodo sensor/actuador (dispositivo final), mantiene únicamente una trayectoria de comunicación directa con el coordinador. Esta topología sencilla se justifica por el reducido número de dispositivos que la conforman (Abbasi et al., 2014). La red configurada trabaja bajo la pila de protocolos ZigBee: un conjunto de protocolos que amplía el estándar IEEE 802.15.4 contemplado para cubrir las necesidades de redes inalámbricas de área personal con velocidades de transferencia baja y reducido flujo de información (Riazahamed, 2009). Las características principales de la red se resumen a continuación: Protocolo de comunicación ZigBee, seis nodos (1 nodo coordinador, 3 nodos sensores y 2 nodos actuadores), topología estrella, banda de frecuencia: ISM de $2,4 \mathrm{GHz}$, canal 15, velocidad de transmisión de 250 Kbps y alcance de 80 a 120m (Yahui et al., 2013).

Nodos de la red. Todos los nodos de la red, incluyen por lo menos un módulo de procesamiento, un módulo de comunicación, un módulo de alimentación eléctrica e interfaces para su conexión (Dargie y Poellabauer, 2010). Si bien, el estándar ZigBee especifica tres dispositivos lógicos nombrados como: Coordinador (ZC), Enrutador (ZR) y Dispositivo Final (ZED), no se agregaron nodos enrutadores (Somani y Patel, 2012).

El ZC está encargado de inicializar la red, establecer el canal de comunicaciones, participar en el encaminamiento de paquetes y ser el origen y/o destinatario de la información. Su módulo de procesamiento está representado por un computador personal (PC) dotado de un software para gestionar todos los datos de la red y definir las acciones de control. La Figura 1, ilustra su composición.

El módulo de comunicación, está constituido por un sistema XBee Pro Series 2 con antena omnidireccional externa. Éste fue configurado para actuar como dispositivo ZC en modo API. Este modo de comunicación, codifica la información en tramas estructuradas y habilita la recepción de muestras remotas (Romero et al., 2015), que corresponden a la humedad volumétrica medida y transmitida por los nodos sensores ubicados en el cultivo. La alimentación eléctrica se garantiza por medio de la conexión al PC por medio del puerto USB.

Cada Nodo Sensor definido como un ZED, también posee como componente principal un módulo XBee Series 2 Pro, que conforma el módulo de comunicación/procesamiento. Su configuración, cumple dos tareas fundamentales: la primera, recibir el voltaje DC entregado por el sensor, digitalizarlo, empaquetarlo y transmitirlo al nodo coordinador cada vez que éste lo solicite. Puesto que la unidad de procesamiento del módulo posee la funcionalidad de un ADC de 10bits para todas sus entradas análogas (Digi International, 2012). La segunda tarea consiste en recibir la señal de control enviada por el ZC en una trama de configuración remota que ordena cambiar el estado del actuador de riego. 


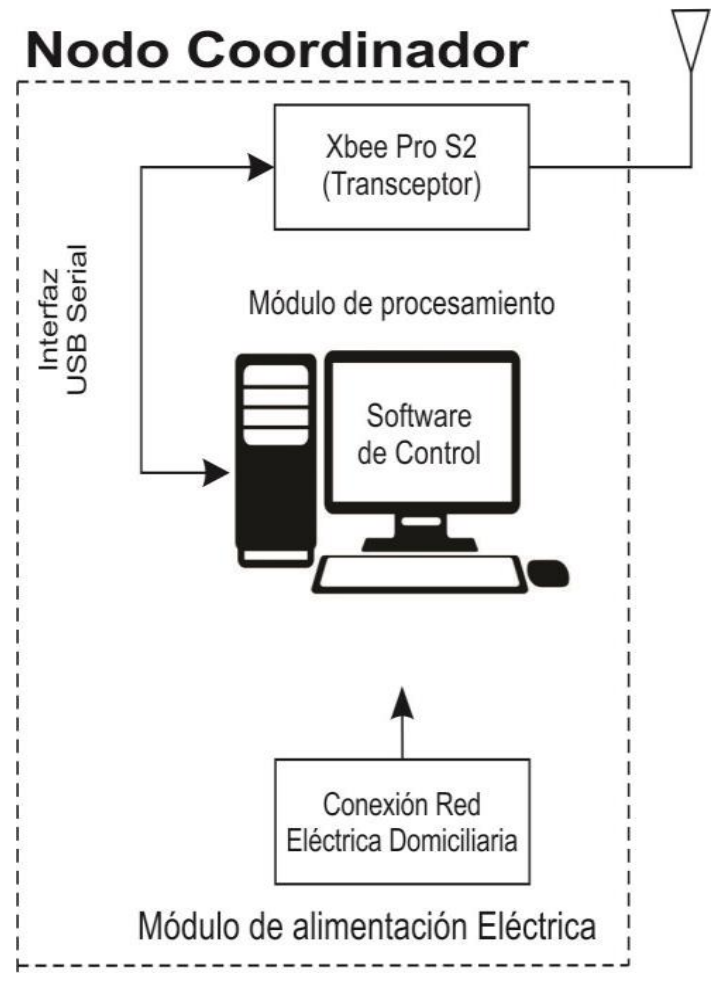

Figura 1. Diagrama de bloques del nodo coordinador.

Este tipo de nodo, puede ser un módulo actuador o un módulo sensor. El primero se encarga de controlar una electroválvula, circuitos de protección para cargas inductivas y acondicionadores de señal para conectarlo a la salida digital del módulo de comunicación/procesamiento. El segundo tipo de nodo, está conectado a un sensor 10HS cuya salida máxima de voltaje es directamente compatible con el máximo voltaje de referencia del ADC del módulo XBee. La Figura 2, ilustra su composición.

Cada Nodo Actuador (también definido como un ZED), funciona únicamente como un receptor de las instrucciones enviadas por el coordinador, que las ejecuta impulsando o frenando el suministro de agua, ya sea para que se efectúe un riego sitio específico por zonas de manejo o un riego generalizado para todo el cultivo (riego tradicional). El nodo actuador NA1 controla una motobomba, mientras que el nodo actuador NA2 controla una electroválvula maestra normalmente cerrada de una pulgada y media de diámetro.
Los dos actuadores, son necesarios, pues, al ser la motobomba, el elemento que impulsa el agua hacia otros cultivos de la finca, era necesario incluir un elemento adicional, una electroválvula maestra, que mantuviera el cultivo de fresas seleccionado, aislado del flujo de agua impulsado cada vez que la motobomba se encendiera para regar otros cultivos. Los módulos son similares a los utilizados en los nodos sensores excepto que no incluyen su módulos homónimos y por tanto, no utiliza ADC así, para el caso de la configuración del módulo de comunicación/procesamiento, el XBee Series 2 Pro, fue habilitado para recibir la señal de control enviada por el coordinador para cambiar el estado de una de sus salida digitales acoplada al actuador (Faludi, 2010). La Figura 3, ilustra su composición.

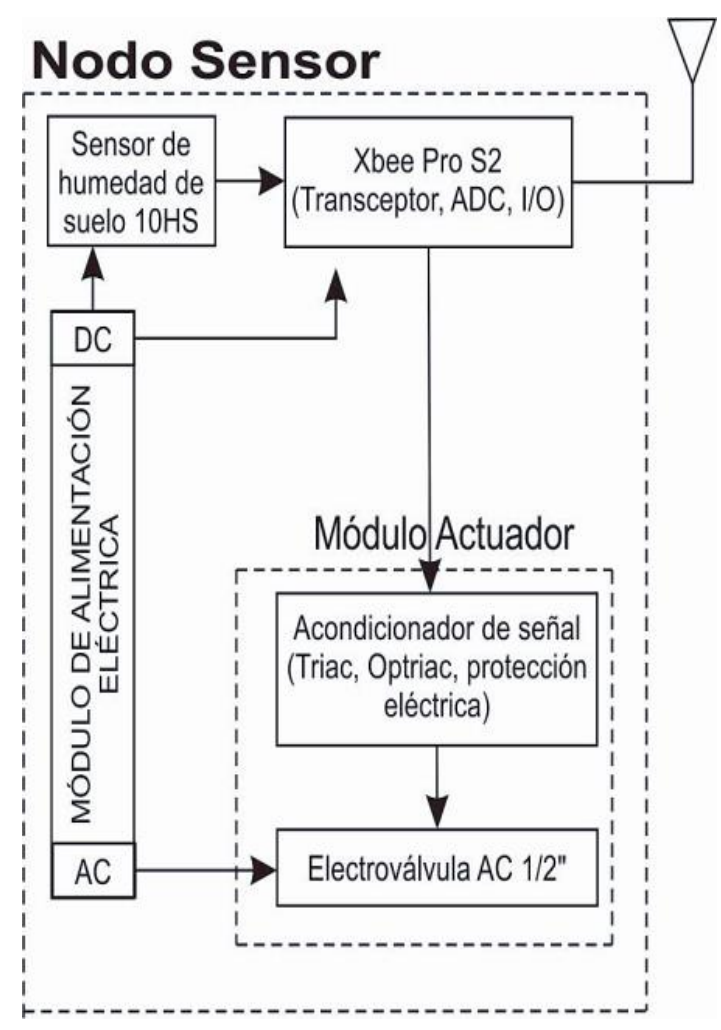

Figura 2. Diagrama de bloques del nodo sensor. 


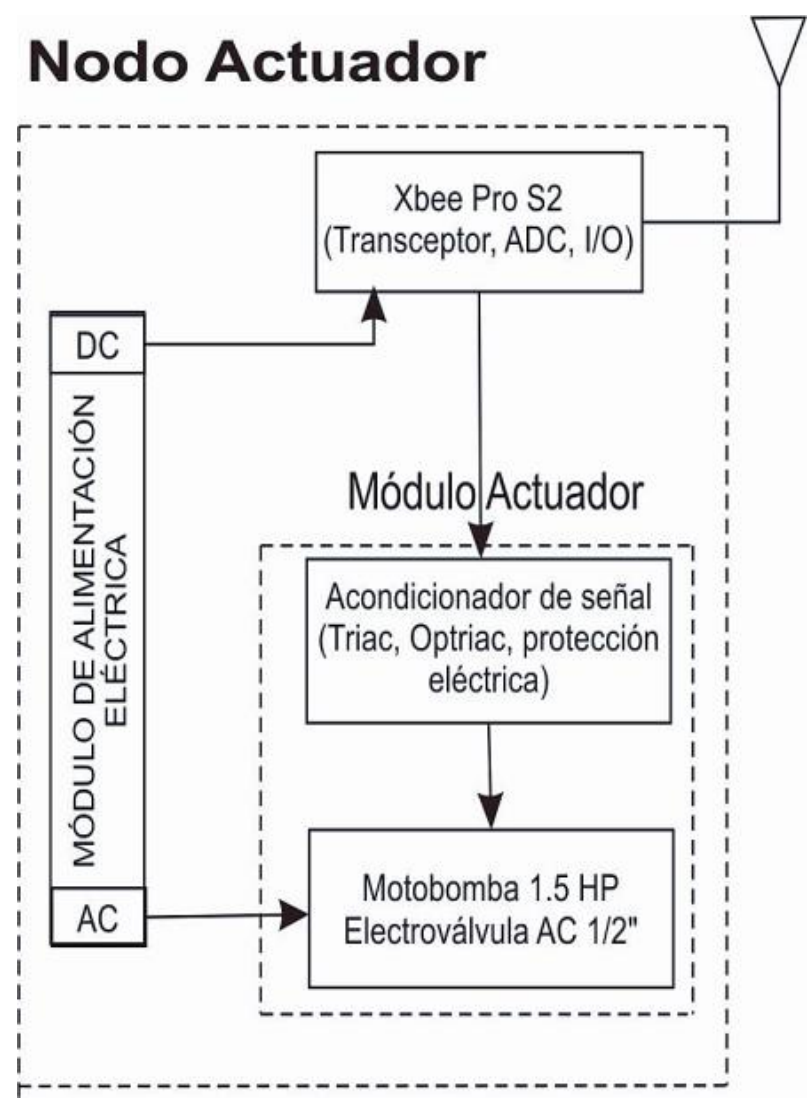

Figura 3. Diagrama de bloques del nodo actuador.

Inicialización y funcionamiento de la red. Una vez configurados los módulos de cada nodo, el coordinador forma la red, asignando aleatoriamente una dirección única de 16bits para identificarla (PAN ID), después, se sintoniza en el canal DSSS 15 y permanece a la espera para asociar nuevos dispositivos. Por otro lado, los dispositivos finales (nodos sensores y actuadores) al ser energizados, buscan en el canal 15 un coordinador y, al detectarlo, se unen a la red, adquieren la misma PAN-ID y se les asigna una dirección de identificación de 16bits para que puedan intercambiar información con el coordinador. Este proceso sucede de forma automática sin intervención del usuario, pero puede verificarse a través de los LEDs indicadores, que brillan de forma intermitente cuando los dispositivos finales están asociados a la red (Digi International, 2012).
Después de la inicialización de la red, los tres nodos sensores miden el nivel de humedad volumétrica que representa su zona de manejo y transmiten el dato inalámbricamente hacia el nodo coordinador. Éste la envía a través de una interfaz serial a un PC provisto del software de control, que despliega los datos a través de una interfaz gráfica, para que el usuario conozca el nivel de humedad individual de cada zona de manejo y la humedad promedio de todo el cultivo.

Cada vez que los datos de humedad ingresan al computador, el software evalúa si el valor está dentro o fuera de los parámetros hídricos permisibles. Dependiendo de los resultados, una señal de control es enviada inalámbricamente para activar 0 desactivar los actuadores que habilitan el paso de agua para aplicar un riego general, o un riego sitioespecifico según la opción definida inicialmente por el usuario.

Software de control. Siendo el software el controlador del sistema de riego, la acción de control elegida fue ON - OFF con banda de histéresis (Ogata, 2009). El algoritmo fue construido en su totalidad en Java, con una interfaz gráfica cuyas funciones incluyen gestionar el puerto serial del PC, almacenar y desplegar el valor de humedad volumétrica y construir y decodificar tramas API (el lenguaje propio de la comunicación con módulos XBee) (Digi International, 2012). Los datos extraídos de las tramas API son evaluados en las ecuaciones de calibración obtenidas experimentalmente para traducir el voltaje del sensor en humedad volumétrica de suelo. Después, los valores de humedad resultantes son analizados para verificar si están dentro de un rango permisible que tiene como límite superior la Capacidad de Campo (CC) y como límite inferior el Umbral de Riego (UR). Estos parámetros hídricos fueron hallados para cada zona de manejo y para todo el cultivo (Carreño y Unigarro, 2005). Finalmente, de acuerdo con el resultado de dicha evaluación, el software construye una nueva trama API que al 
ser transmitida, configura el estado de los nodos remotos para que sus actuadores se activen o desactiven haciendo el control de riego.

Implementación. Los circuitos impresos, fueron diseñados con la herramienta EAGLE considerando la potencia disipada por los módulos. Todos los componentes de los nodos sensores $\mathrm{y}$ actuadores fueron protegidos utilizando cajas herméticas para intemperie y ajustados en soportes elevados para lograr línea de vista con el nodo coordinador. La Figura 4 muestra algunas imágenes de éste procedimiento.

El archivo ejecutable del software de control y la máquina virtual de Java fueron instalados en el PC para que el usuario ejecutara en cualquier momento la interfaz gráfica. Para el caso de los nodos sensores, éstos fueron ubicados a una distancia entre 70 y $100 \mathrm{~m}$ respecto al nodo coordinador; cada uno en la parte central de un surco guía y todos los sensores fueron enterrados verticalmente a una profundidad de $20 \mathrm{~cm}$ del suelo justo bajo un emisor de una cinta de goteo.

El nodo actuador NA1 fue ubicado contiguo a la motobomba a $30 \mathrm{~m}$ del nodo coordinador. Su módulo actuador integra un circuito de potencia robusto y un sistema de protección que involucra una red snubber y un disyuntor termo-magnético por el alto amperaje demandado. El circuito de potencia fue construido con un ancho de pista cercano a $1 \mathrm{~cm}$ y provisto de un disipador de aletas proporcional a la energía disipada por el semiconductor conmutador (Biswanath, 2014; Littelfuse, 2008). El nodo actuador NA2 fue ubicado a la salida del cabezal de riego lateral al cultivo, para conectar fácilmente la electroválvula maestra a la tubería principal. Su caja hermética incluye los circuitos modulares y una fuente DC para su alimentación y la de los tres nodos sensores.

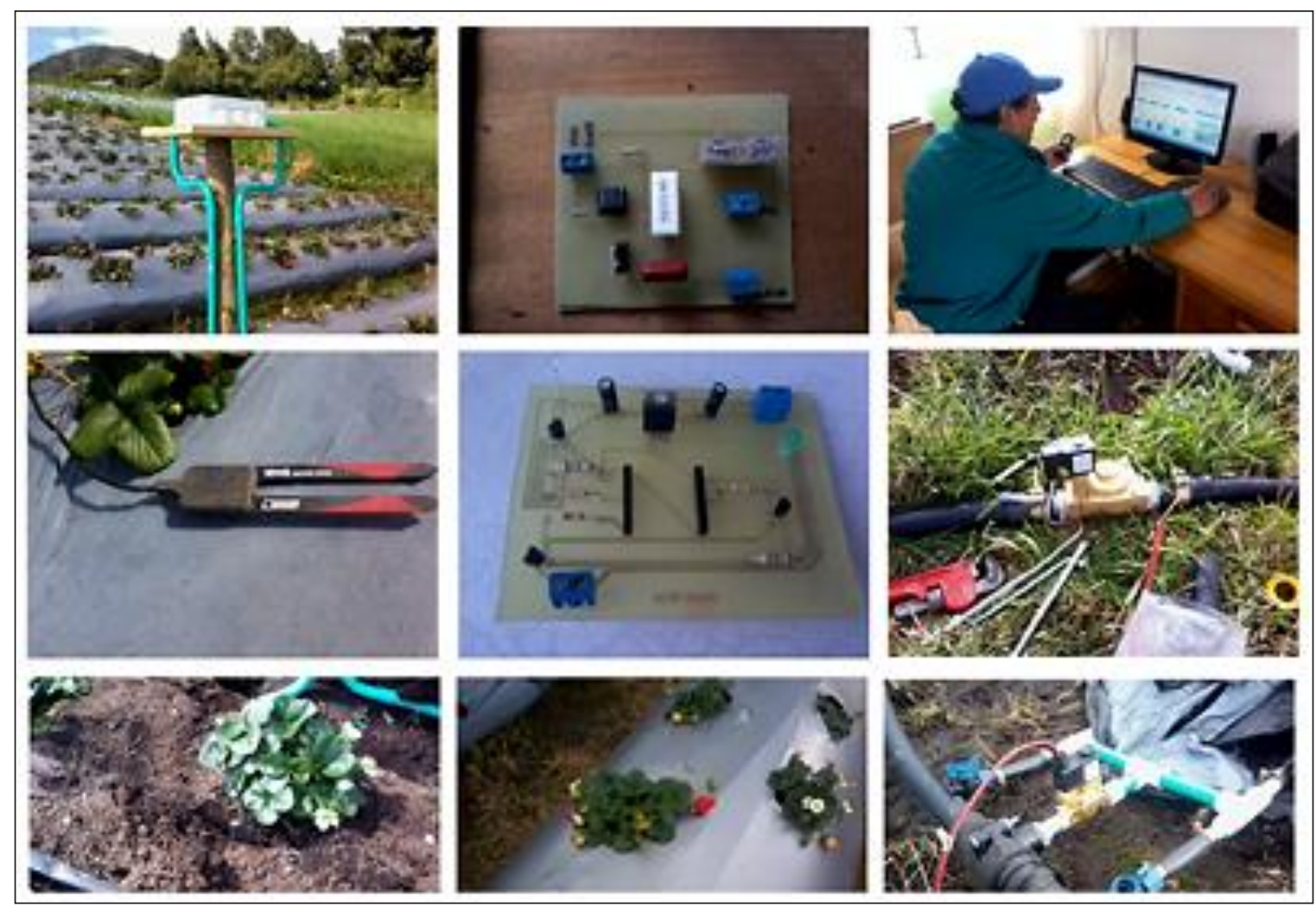

Figura 4. Implementación del nodo sensor. 


\section{RESULTADOS Y DISCUSIÓN}

Sistema prototipo. El prototipo diseñado, es un sistema de control de lazo cerrado en el que la variable de salida del proceso (la humedad volumétrica del suelo) se mide y se retroalimenta al controlador, que determina el error de la medida con su valor de referencia y genera una acción que ejecutan los actuadores. El elemento esencial del sistema de control, resulta ser el controlador, pues está encargado de detectar los desvíos del valor medido por los sensores fuera del rango de humedad permisible y emitir una señal de corrección hacia los actuadores, para que se produzca la acción de control establecida que, para éste caso, es la acción de control ON - OFF con banda de histéresis. La Figura 5 muestra el diagrama de bloques del sistema.

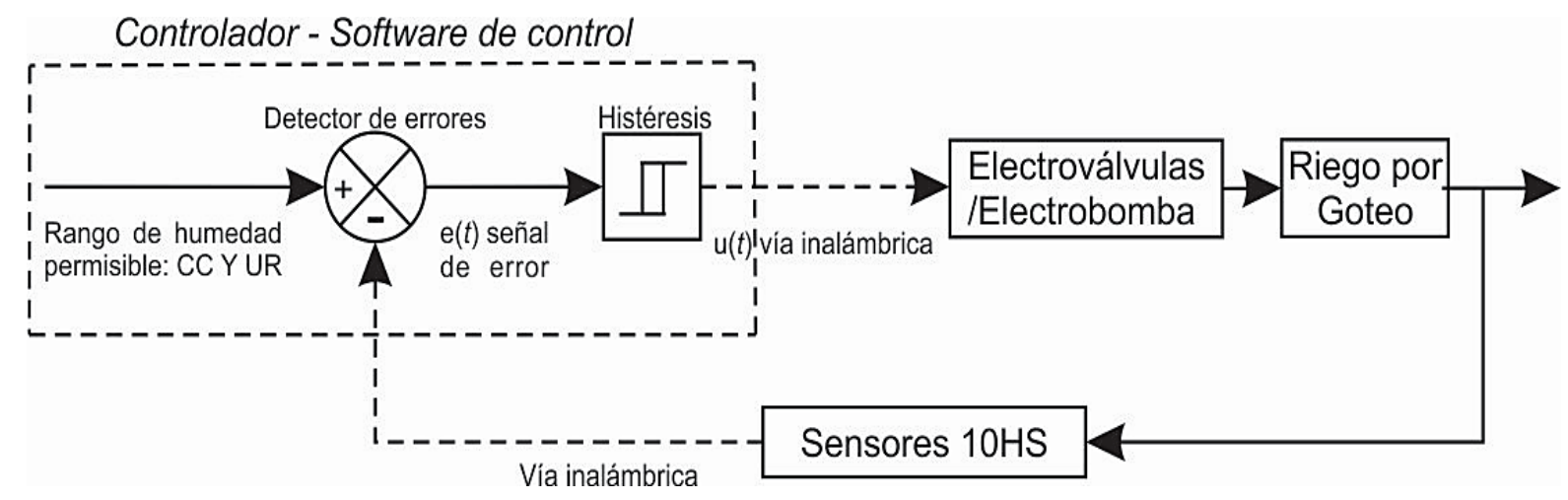

Figura 5. Sistema Prototipo para la automatización y control del riego localizado

Riego sitio - específico. Una vez configurada la opción de riego surco a surco o sitio-especifico, el proceso se inicializa y los nodos de la WSN empiezan a funcionar intercambiando datos. En la interfaz gráfica, se despliega el nivel de humedad de cada surco guía. Inicialmente todos los surcos se encuentran por fuera del rango de humedad per- misible, por lo que todas las electroválvulas se activan automáticamente para regar su respectiva zona de manejo. El sistema, permaneció activo durante 20 minutos aproximadamente después de su inicialización, tiempo en el cual todos los surcos guía alcanzaron su capacidad de campo en instantes distintos. La Tabla 2 muestra los resultados.

Tabla 2. Resultados riego sitio - específico aplicado a cada zona de manejo del cultivo de acuerdo a su nivel de humedad.

\begin{tabular}{lcccc}
\hline Surco guía & $\begin{array}{c}\text { Rango } \\
\text { permisible } \\
(C C, \mathrm{HR}) \%\end{array}$ & $\begin{array}{c}\text { Valor } \\
\text { inicial } \\
\theta_{\boldsymbol{v}}(\%)\end{array}$ & $\begin{array}{c}\text { Tiempo } \\
\text { establecimiento } \\
{ }_{t_{r}(\mathrm{~min})}\end{array}$ & $\begin{array}{c}\text { Valor } \\
\text { final } \\
\theta_{\boldsymbol{v}}(\%)\end{array}$ \\
\hline Zona Alta & $(40,10,37,40)$ & 28,9 & $10 \mathrm{~min}$ & 42,4 \\
Zona Media & $(39,75,37,07)$ & 31,6 & $17 \mathrm{~min}$ & 40,8 \\
Zona Baja & $(42,05,39,21)$ & 32,8 & $19 \mathrm{~min}$ & 42,7 \\
\hline
\end{tabular}


Es evidente que el cultivo presenta una variabilidad de humedad de suelo a través de los tres surcos analizados. Se observa que, al encender el sistema, las humedades son distintas en todos los surcos, siendo el surco guía de la zona de manejo Alta el que presenta una menor humedad, pero siendo el que menor tiempo de riego requiere, pues adquiere rápidamente su capacidad de campo.

Riego tradicional. Configurado el riego para actuar en modo de Riego Tradicional por primera vez, la interfaz gráfica mostró un bajo nivel de humedad volumétrica promedio en todo el cultivo, razón por la cual, el riego se activa inmediatamen- te para todos los surcos encendiendo la motobomba y la electroválvula maestra. De igual manera, el sistema permanece activo durante 20 minutos antes de alcanzar su capacidad de campo promedio. La Tabla 3 muestra los resultados para este caso.

Considerando la humedad volumétrica de todo el cultivo como un valor único promedio, los resultados, muestran que dicho valor no presenta diferencias excesivas a aquellas medidas de forma independiente en cada surco guía o en sí, de cada zona de manejo. En cuanto al tiempo de riego, tampoco expresan cambios bruscos, excepto si se compara con el tiempo de riego de la zona alta.

Tabla 3. Resultados riego tradicional aplicado a todo el cultivo de acuerdo a su humedad promedio.

\begin{tabular}{lcccc}
\hline Surco guía & $\begin{array}{c}\text { Rango } \\
\text { permisible } \\
(C C, \mathrm{UR}) \%\end{array}$ & $\begin{array}{c}\text { Valor } \\
\text { inicial } \\
\theta_{v}(\%)\end{array}$ & $\begin{array}{c}\text { Tiempo } \\
\text { establecimiento } \\
t_{r}(\min )\end{array}$ & $\begin{array}{c}\text { Valor } \\
\text { final } \\
\theta_{v}(\%)\end{array}$ \\
\hline Cultivo Total & $(39,97,37,27)$ & 30,6 & 19 & 40,7 \\
\hline
\end{tabular}

\section{CONCLUSIONES}

El diseño de una WSN, obtiene los datos de humedad volumétrica en un cultivo de fresas y permite controlar, de forma general o sitio-específica, el riego por goteo en el cultivo. Se describieron todos los componentes implementados en el prototipo, el cual hizo posible verificar la existencia de un grado de variabilidad espacial de humedad de suelo cultivable. De esta forma, se evidencia la importancia del uso de herramientas tecnológicas para lograr una caracterización de humedad de suelo promedio mucho más precisa, definir bajo criterios técnicos cuánto y cuándo regar en diferentes áreas del cultivo.

\section{AGRADECIMIENTOS}

Este trabajo fue posible gracias a la financiación de la Fundación Social "Pasto - Colombia". Los autores agradecen especialmente a Guillermo Torres, director regional de esta organización.

\section{REFERENCIAS BIBLIOGRÁFICAS}

ABBASI, A. Z., ISLAM, N., SHAIKH, Z. A. 2014. A review of wireless sensors and networks' applications in agriculture. Computer Standards \& Interfaces. 36(2):263-270 p.

BISWANATH, P. 2014. Industrial Electronics and Control. PHI Learning Pvt. Ltd. 227 - 324 p.

BADILLO, M. F., VALDERA, F., BODAS, V., FUENTELSAZ, F., PEITEAD0, C., \& WWF, E. 2009. Manual de buenas prácticas de riego, Propuestas de WWF para un uso eficiente del agua en la agricultura. En: http://awsassets. wwf. es/ downloads/buenas_practicas_de_riego. Pdf; consultado : agosto, 2016.

CARREÑO, M. R.; UNIGARRO. A. 2005. Metodología para el análisis físico de suelos. En: Métodos químicos para el análisis de suelos. Universidad de Nariño. 56 - 57 p.

COBOS, D.; CHAMBERS, C. 2010. Calibrating ECH20 soil moisture sensors. Application Note, En: Decagon Devices, http://manuals.decagon.com/Application $\% 20$ Notes/13393_Calibrating\%20ECH20\%20Probes_Print. pdf; consulta: agosto, 2016. 
DARGIE, W.; POELLABAUER, C. 2010. Fundamentals of Wireless Sensor Networks. Theory and Practice. First edition. Wiley. Chennai, India. 48 - 61 p.

DEAQUIZ, Y.; ÁLVAREZ-HERRERA, J.; PINZÓN-GÓMEZ, L. 2014. Efecto de diferentes láminas de riego sobre la producción y calidad de fresa (Fragaria sp.). Revista Colombiana de Ciencias Hortícolas. 8(2):192 - 205.

DECAGON, D. 2009. 10HS Soil Moisture Sensor Operator's Manual, Version 2.0. Decagon Devices Inc. 21 p.

DIGI INTERNATIONAL. 2012. XBee/XBee PRO ZB RF Modules. En: https://www.digi.com/support/ productdetail?pid=3430; consulta: agosto, 2016.

FALUDI, R. 2010. Building wireless sensor networks: with ZigBee, XBee, arduino, and processing. O'Reilly Media. $322 \mathrm{p}$.

GONZÁLEZ R. F., HERRERA P. J., HERNÁNDEZ B. O., LÓPEZ S. T., CID L. G. 2012. Base de datos sobre necesidades hídricas. Revista Ciencias Técnicas Agropecuarias. 21(2):42 - 47.

KLOHN, W;; APPELGREN, B. 1999. Agua y Desarrollo. Revista CIDOB d'Afers Internacionals. 45:105 - 126.

KUNCHAM, V. S., RAO, N. V. 2014. Sensors for Managing Water Resources in Agriculture. IOSR Journal of Electronics and Communication Engineering (IOSR-JECE). 9(2):145 - 163.

LITTELFUSE. 2008. Thyristors Used as AC Static Switches and Relays. En: littelfuse application_notes. En: http:// www.littelfuse.com/ /media/electronics_technical/ application_notes/switching_thyristor/littelfuse_thyristors_used_as_ac_static_switches_and_relays_application_ note.pdf; consulta: agosto, 2016.

MANDAL, K.; MAITY, A. 2013. Precision Farming for Small Agricultural Farm: Indian Scenario. American Journal of Experimental Agriculture. 3:200 - 217.

MATHWORKS. 2016. Data Fitting with Matlab. En: Data Fitting with MATLAB, http://www.mathworks.com/discovery/data-fitting.html; consulta: agosto, 2016.

MONTAVANI, E. C.; PINTO, F. A. C.; QUEIROZ, D. N. 2006. Introducción a la Agricultura de Precisión. 15 - 21 p. En: Agricultura de Precisión: Integrando Conocimientos para una Agricultura Moderna y Sustentable. Procisur/IICA. Montevideo.
MUÑOZ, R.; RITTER, A. 2005. Medida de la humedad del suelo. En: Hidrología agroforestal. Mundi-Prensa. 127 $133 \mathrm{p}$.

OGATA, K. 2009. Ingeniería de Control Moderna. Quinta edición. Prentice Hall. 17 - 29 p.

RIAZAHAMED, S. 2009. The Role of ZigBee Technology in Future Data Communication System. Journal of Theoretical and Applied Information Technology. 5:113 - 118 p.

ROEL, A.; TERRA, J. 2006. Muestreo de Suelos y Factores Limitantes del Rendimiento. En: Agricultura de Precisión: Integrando Conocimientos para una Agricultura Moderna y Sustentable. Procisur/IICA. Montevideo. 67 - 80 p.

ROMERO, V., ALBERTO, C., JAIMES, B., ERICKSON, J., PABÓN, D. C. 2015. Configuration Parameters in Module XBEE-PRO ${ }^{\circ}$ ZB S2B for Measuring Environmental Variables. Tecnura. 19(45):141 - 157.

SAC. SOCIEDAD DE AGRICULTORES DE COLOMBIA. 2010. Una locomotora que impulsa el desarrollo del país. Revista Nacional de Agricultura. 956:4 - 6 p.

SOHRABY, K., MINOLI, D.; ZNATI, T. 2007. Wireless sensor networks: technology, protocols, and applications. John Wiley \& Sons. 326 p.

SOMANI, N. A.; PATEL, Y. 2012. ZigBee: A Low Power Wireless Technology for Industrial Applications. International Journal of Control Theory and Computer Modelling, 2:27 - $33 \mathrm{p}$.

SPELMAN, D., KINZLI, K. D., KUNBERGER, T. 2013. Calibration of the 10HS Soil Moisture Sensor for Southwest Florida Agricultural Soils. Journal of Irrigation and Drainage Engineering. 139(12):965 - 971.

VEGA, J. A., RUIZ, M. P. 2013. Agricultura de precisión: hacia la integración de datos espaciales en la producción agraria. En: http://www.mapama.gob.es/ministerio/pags/Biblioteca/Revistas/pdf_AM/Ambienta_2013_105_16_27.pdf; consulta: junio, 2015.

YAHUI, W., LEIPENG, Y., PANGUO, F. 2013. Design of Oilfield Remote Monitoring System Based on ZigBee Technolgy. Computer Measurement \& Control. 2:028 p.

ZHANG, N.; WANG, M.; WANG, N. 2002. Precision Agriculture - A Worldwide Overview. Computers and Electronics in Agriculture. 36:113 - 132 p. 